\title{
PLANIFICACIÓN DEL SISTEMA DE EVALUACIÓN DEL DESEMPEÑO DE RECURSOS HUMANOS: EL CASO DE EMPRESAS CHILENAS
}

\author{
PLANNING OF EVALUATION SYSTEM OF PERFORMANCE OF HUMAN \\ RESOURCES. THE CASE OF CHILEAN COMPANIES
}

\section{PLANEJAMENTO DO SISTEMA DE AVALIAÇÃO DO DESEMPENHO DOS RECURSOS HUMANOS: O CASO DAS EMPRESAS CHILENAS}

\section{Recebimento: 01/03/2018- Aceite: 05/08/2018- Publicação: 28/10/2018 Processo de Avaliação: Double Blind Review}

\section{Jorge Sánchez Henríquez.}

Escuela de Auditoria e Ingeniería en Control de Gestión, Facultad de Economía y Negocios, Universidad de Talca, Chile

jsanchez@utalca.cl

\section{Sándra Alvear Vega}

Escuela de Auditoria e Ingeniería en Control de Gestión, Facultad de Economía y Negocios, Universidad de Talca, Chile

salvear@utalca.cl

\section{RESUMEN}

El objetivo principal de este trabajo es identificar las variables que influyen en la evaluación de la etapa "planificación" en el proceso de medición del desempeño de los trabajadores y proponer un modelo que permita estimar el efecto que produce cada variable en la evaluación de dicha etapa. Es un estudio analítico, observacional de cohorte transversal de 90 empresas chilenas, públicas y privadas, de diferentes rubros. Los resultados del análisis de la regresión lineal múltiple sugieren que las variables con valor predictivo al momento de evaluar la etapa de "planificación" son: objetivo, existencia, orientación, cumplimiento, calidad e integridad. Esta línea de investigación se puede continuar contrastando lo planificado con la implementación final del proceso de evaluación que realizan las empresas.

\footnotetext{
${ }^{1}$ Autor para correspondência: Escuela de Auditoria e Ingeniería en Control de Gestión, Facultad de Economía y Negocios, Universidad de Talca, Chile. Casilla 721 Talca. Avenida Lircay s/n Talca. Chile.
} 
Palabras clave: Auditoria de recursos humanos, evaluación del desempeño y planificación de la evaluación del desempeño.

\begin{abstract}
The objective of this paper is to identify the main determinants of the planning stage in the process of performance evaluation of employees. Moreover we propose a model to estimate the impacts of these factors. Ninety public and private Chilean firms--belonging to different economic sectors, participate in an analytical and cohort study. Results of multiple linear regression suggest that the factors with predictive power are goal, existence, orientation, fulfillment, quality, and integrity. To continue contrasting planned vs. implemented actions into the performance evaluation is a live of research that is suggested by this study.
\end{abstract}

Key words: Human Resources Audit, Performance evaluation, Performance evaluation planning stage

\title{
RESUMO
}

O principal objetivo deste trabalho é identificar as variáveis que influenciam a avaliação do estágio de "planejamento" no processo de mensuração do desempenho dos trabalhadores e propor um modelo que permita estimar o efeito produzido por cada variável na avaliação do referido estágio. . Trata-se de um estudo de coorte transversal analítico, observacional, com 90 empresas chilenas, públicas e privadas, de diferentes áreas. Os resultados da análise de regressão linear múltipla sugerem que as variáveis com valor preditivo ao avaliar o estágio de "planejamento" são: objetivo, existência, orientação, conformidade, qualidade e integridade. Esta linha de pesquisa pode ser continuada, contrastando o planejado com a implementação final do processo de avaliação realizado pelas empresas.

Palavras-chave: Auditoria de recursos humanos, avaliação de desempenho e planejamento de avaliação de desempenho.

\section{INTRODUCCIÓN}

Uno de los motivos más importantes por el que las organizaciones deben implementar un sistema de evaluación y control de gestión de su personal, es para saber si sus trabajadores están efectivamente contribuyendo al logro de los objetivos establecidos. Pereda, Berrocal y López (2002), Gruman y Saks (2011) y Kehoe y Wright (2013) plantean que contar con profesionales adecuados implica que tengan un desempeño exitoso y que estén comprometidos con la organización. Para Pérez, Leyva, Bajuelo y Pérez (2015) la evaluación del desempeño es un subsistema de gran importancia dentro de la gestión del capital humano, al ser uno de los principales mecanismos de control de los resultados y puede desarrollarse en los diferentes niveles de la entidad. Las personas en las empresas hoy se constituyen en un recurso muy relevante, lo relevante es que los ejecutivos de 
recursos humanos comprendan que las personas son el principal activo de una compañía y que trabajadores contentos, motivados y comprometidos es sinónimo de mayor productividad. Para Silva, Oliveira, Rosini \& Silva (2016) el resultado de las organizaciones se relaciona con las mejoras continuas en el desempeño y la productividad de los trabajadores. Por su parte (Parent, Sloan, y Tsuchida, 2015, p. 56), plantean que si no se maneja cuidadosamente, la gestión del desempeño puede no sólo desperdiciar tiempo valioso, sino también tener un efecto negativo sobre el compromiso y la retención. Si se maneja bien, puede ser uno de los eventos de desarrollo más inspiradores en la carrera de un empleado, y puede impulsar mejoras en desempeño y resultados a nivel de toda la organización. Para Sastre y Aguilar (2003), Kliksberg (1998), Valle (2004) y Bock (2015) las organizaciones deben evaluar el desempeño de sus trabajadores si quieren asegurarse realmente de cumplir con sus objetivos estratégicos, en efecto, la mayoría de las empresas reconocen que el personal es un componente fundamental de su negocio. En este sentido, Wright, Dunford y Snell (2001), citados por Ramlall (2006), hicieron énfasis en la importancia que tiene para las organizaciones contar con prácticas de recursos humanos que contribuyan a obtener un alto desempeño y que posicionen a las personas como fuente de ventaja competitiva. En efecto si bien siempre se evalúa el desempeño, a medida que las organizaciones se desarrollan necesitan implementar sistemas que les entreguen datos confiables y válidos respecto al desempeño de sus trabajadores, ya que existen diversas decisiones que deben tomar relacionadas precisamente con sus trabajadores y que para que sean efectivas necesitan información relevante en el momento oportuno. Para Manjarrés, Castell y Luna (2013) la evaluación del desempeño tiene que ir alineada con el direccionamiento estratégico de la empresa, los procesos organizacionales y con los objetivos e indicadores que se quieren lograr a lo largo de la operación organizativa.

Las personas encargadas de la gestión de recursos humanos ocupan hoy un lugar central en las organizaciones, debido a que son los individuos los que influyen directamente en la eficiencia de los procesos y por ende en los resultados organizacionales (Maristany, 2000 y Exley y Legge 2001). En ese contexto este trabajo es fruto de una línea de investigación relacionada con la gestión y el control del desempeño, como así también con auditoría de recursos humanos. 


\section{MARCO TEÓRICO}

\subsection{AUDITORÍA Y RECURSOS HUMANOS}

La auditoría de recursos humanos es un proceso de evaluación sistemático que validará las políticas, prácticas y programas de recursos humanos (Hernández, 2007), se orienta a evaluar la gestión estratégica de los recursos humanos (De Quijano y Navarro, 1999; Nevado, 1988 y Sánchez y Bustamante, 2008), la auditoría de la gestión de recursos humanos abarcan a un triple colectivo; los especialistas en recursos humanos, los directivos de línea y los trabajadores (Alfrado de Prado, 1999), por tanto una auditoría del equipo directivo, concierne no solamente a la organización interna de un equipo de dirección, sino también a sus valores, a su estilo de dirección y sus orientaciones (García, 2003), la auditoría de recursos humanos es la que suministra a la organización datos sobre los niveles operacional, gerencial y estratégico (Fombrum, Tichy y Devanna, 1984), en definitiva las auditorías de recursos humanos, examinan si se realizan y siguen las políticas y prácticas de recursos humanos (Milkovich y Boudreau, 1994), la auditoría de recursos humanos es un procedimiento que tiene por objeto revisar y comprobar, bajo un método especial, todas las funciones y actividades de personal (Reyes, 2002), su ámbito de acción es más amplio ya que se refiere no solamente a la formación interna de un equipo de dirección, sino también a sus valores, a su estilo de dirección y sus orientaciones (García, 2003), se buscan deficiencias y se ayuda a mejorar, así como también mejorar a cada uno de los trabajadores de la organización (Pérez y Oreo, 2006), al realizar una auditoría de recursos humanos se obtienen beneficios y se verifican aspectos importantes para la organización (Werther y Davis, 2008), en esa misma línea va Gonzalez (2010), al enfrentar la auditoría de recursos humanos en el proceso de compensaciones al personal.

Uno de los primeros programas de auditoría publicados aunque muy rudimentario es de origen brasileño (Siegfried,1965), en la misma línea hay un clásico que en sus inicios se atrevió a diseñar un programa de auditoria (Chiavenato, 2007), claramente hay una gran dificultad en el diseño de un programa de auditoría para evaluar recursos humanos y ello se debe al cruce de dos áreas muy diferentes la auditoria y los recursos humanos, una adecuada evaluación por tanto, debe considerar ambos marcos teóricos, en efecto, la construcción del programa de auditoría publicado por Sánchez y Rojas (2014), posee como soporte teórico ambos marcos, uno que viene desde la vertiente de la auditoría clásica con autores tales 
como Paton (1943), Mautz (1970), Coopers y Lybrand (1984), Slosse y otros (1991), Arens y Loebbecke (1996), Téllez (2004), Whittington y Pany (2005), Arens, Elder y Beasley (2007), PriceWaterhouseCoopers (2007) y Fonseca (2013). El otro marco proviene desde el área de los recursos humanos con autores como Miranda, Torras y González (1982), Bentley (1993), Valle y Weiss (1995), Rodriguez y Ramirez (1997), Dolan, Schuler y Valle (1999), Reyes (2002), Sastre y Aguilar (2003), Ariza, Morales y Morales (2004), Mondy y Noe (2005), Meza, Naranjo y Pérez (2007), Chiavenato (2007), Werther y Davis (2008) y Sánchez (2013).

\subsection{EVALUACIÓN DEL DESEMPEÑO}

Para Pereda, Berrocal y López (2004), la evaluación del desempeño es el proceso sistemático y periódico de medida objetiva del nivel de eficacia y eficiencia de un empleado, o equipo, en su trabajo, Gómez-Mejía, Balkin y Cardy (2001) complementan diciendo que la valoración del rendimiento implica identificar, medir y gestionar el rendimiento de las personas de una organización. Por su parte Sastre y Aguilar (2003) postulan que es un proceso sistemático y estructurado, de seguimiento de la labor profesional del empleado, para valorar su actuación y los resultados logrados en el desempeño de su cargo. Ariza, Morales y Morales (2004) complementan afirmando que el desempeño es el grado en el cual los empleados cumplen con los requisitos del trabajo. Partiendo de esta premisa, la evaluación del desempeño es un proceso de valoración del desempeño de los trabajadores. Para Sánchez, Pizarro, Alvarez, Castillo \& Alfaro (2017) existe una importancia conceptual y práctica de la compensación, a nivel de variable relevante al momento de gestionar el desempeño del personal. Siguiendo con el análisis del desempeño Dessler y Varela (2004) definen la evaluación como la valoración del desempeño actual o anterior de un trabajador en comparación con estándares, en el mismo sentido Mondy y Noe (2005) lo asocian a un sistema de revisión y evaluación del desempeño laboral individual o de equipos, mientras que Sánchez y Bustamante (2008), lo relacionan con valor agregado y plantean que las organizaciones necesitan conocer como están desempeñando sus labores los empleados, a fin de identificar quienes efectivamente agregan valor y cuáles no, estos autores van mucho más allá al plantear que hay que discriminar entre empleados efectivos e inefectivos. 
A medida que ha avanzado el tiempo las organizaciones han internalizado que el factor clave que determina su éxito es la forma en que aprovechan el trabajo de sus empleados, es por esto, que con el paso del tiempo también se han dado cuenta que más que empleados, son recursos para la organización, de ahí que surge el término "recursos humanos". De esta forma nace la necesidad de saber cómo se están aprovechando estos recursos y si los mismos están aportando a la organización y para esto es necesario evaluar su desempeño. Sin embargo, como mencionan Sánchez y Calderón (2012) la evaluación históricamente se restringió al simple juicio unilateral del jefe respecto al trabajo de su empleado pero a medida que ha evolucionado la gestión de recursos humanos, se han establecido generaciones de modelos de evaluación hasta el punto que hoy en día se pueden encontrar modelos de evaluación de cuarta generación. La evaluación del desempeño, cuenta con visiones más contemporáneas como las planteadas por diversos autores tales como Gomez-Mejia, Balkin y Cardy (2001), Sastre y Aguilar (2003), Ariza, Morales y Morales (2004), Dessler y Varela (2004), Pereda, Berrocal y López (2004), Mondy y Noe (2005) y Sanchez (2013). Para Salgado (2011) la evaluación del desempeño tiene el propósito de valorar de forma sistemática y objetiva, el rendimiento o desempeño de los empleados en la organización. Es el grado en que el empleado cumple con los requisitos de su cargo (Trillo, 2001) o el modo en que un trabajador realiza las funciones y tareas que tiene asignadas, (Salgado y Cabal, 2011), por su parte Valdés, Garza, Pérez, Gé y Chávez (2015) complementan diciendo que la evaluación del desempeño debe estar de acuerdo con la misión y los objetivos fijados por la empresa, y demuestra en el ejercicio de sus funciones poseer las competencias exigidas para el puesto de trabajo que ocupa el trabajador.

\subsection{PLANIFICACIÓN DEL PROCESO DE EVALUACIÓN DEL DESEMPEÑO}

No sólo es importante definir formalmente el marco referencial, a través de una planificación del proceso, sino que esa planificación debe sujetarse estrictamente a los parámetros de dicho marco referencial-conceptual, en efecto todas las directrices estratégicas del proyecto deben acotarse en relación a dicho marco, en esa línea Manjarrés (2013) es enfático al afirmar que un modelo de evaluación del desempeño guiará a la empresa a conocer cómo influye cada uno de los empleados en la consecución de los 
objetivos organizacionales. Para Luecke (2007), lo primero a realizar es planificar las metas relacionadas con el desempeño del trabajador, sin embargo existen aspectos importantes dentro del proceso que es necesario considerar, en efecto Fernández, Cubiero y Dalziel (1996), mencionan que los pasos son los siguientes: a) determinar con claridad la razón por la que se desea implementar el proceso. La organización tiene que decidir cuáles son los críticos resultados que necesita obtener por medio del proceso. b) diseñar un proceso adaptado a esas finalidades: Es muy probable que dentro de una misma organización el proceso tenga que variar a fin de reflejar las distintas funciones y puestos de trabajo. c) proporcionar una adecuada formación a todo el personal que interviene el proceso y d) examinar continuamente el impacto del proceso. Para Luecke (2007) lo más relevante es identificar los problemas en el desempeño que tienen los trabajadores y eliminarlos, en la misma línea están (Sánchez y Rojas, 2014, p. 418) quienes plantean que en esta etapa la empresa establece de antemano qué hará, para alcanzar sus objetivos, el proceso de planificación se inicia desde el momento en que la empresa comienza a buscar la información base para la planeación, es decir, aquella información que puede satisfacer el surgimiento de la necesidad de evaluar el desempeño, en este sentido, se debe cumplir con las siguientes actividades: Búsqueda de información, planteamiento de objetivos, identificación de riesgos potenciales que impidan el cumplimiento de su objetivo, toma de decisiones de los aspectos principales sobre los que se basará el proceso, establecer las etapas del proceso y las actividades, secuencia y estimación de tiempo, planificación de recursos (qué recursos, cantidad y cuánto tiempo), programación de actividades, asignación de recursos, elaboración de documento formal, programación de las actividades, asignación de recursos y elaboración de un documento formal.

\section{MATERIAL Y MÉTODOS}

El presente trabajo tiene como objetivo identificar las variables que influyen en la evaluación de la etapa "planificación" del proceso de medición del desempeño de los trabajadores en diversas organizaciones y proponer un modelo que permita estimar el efecto que produce cada variable en combinación con las demás en la planificación del desempeño en las organizaciones.

Es un estudio analítico, observacional de cohorte transversal de 90 empresas chilenas, públicas y privadas, de diferentes rubros, a nivel de tamaño de las empresas ellas 
se distribuyen en 38 Grandes, 12 Medianas, 24 Pequeñas y 6 Microempresas. Los datos fueron recopilados entre el 10 de abril de 2015 y el 30 de septiembre de 2016, a partir de un programa de auditoría que incluyó la aplicación de 27 procedimientos de auditoria. Estos procedimientos se aplican a todo el proceso de planificación según lo indicado en la Figura 1 .

Figura 1: Flujograma del proceso de planificación del desempeño en la organización

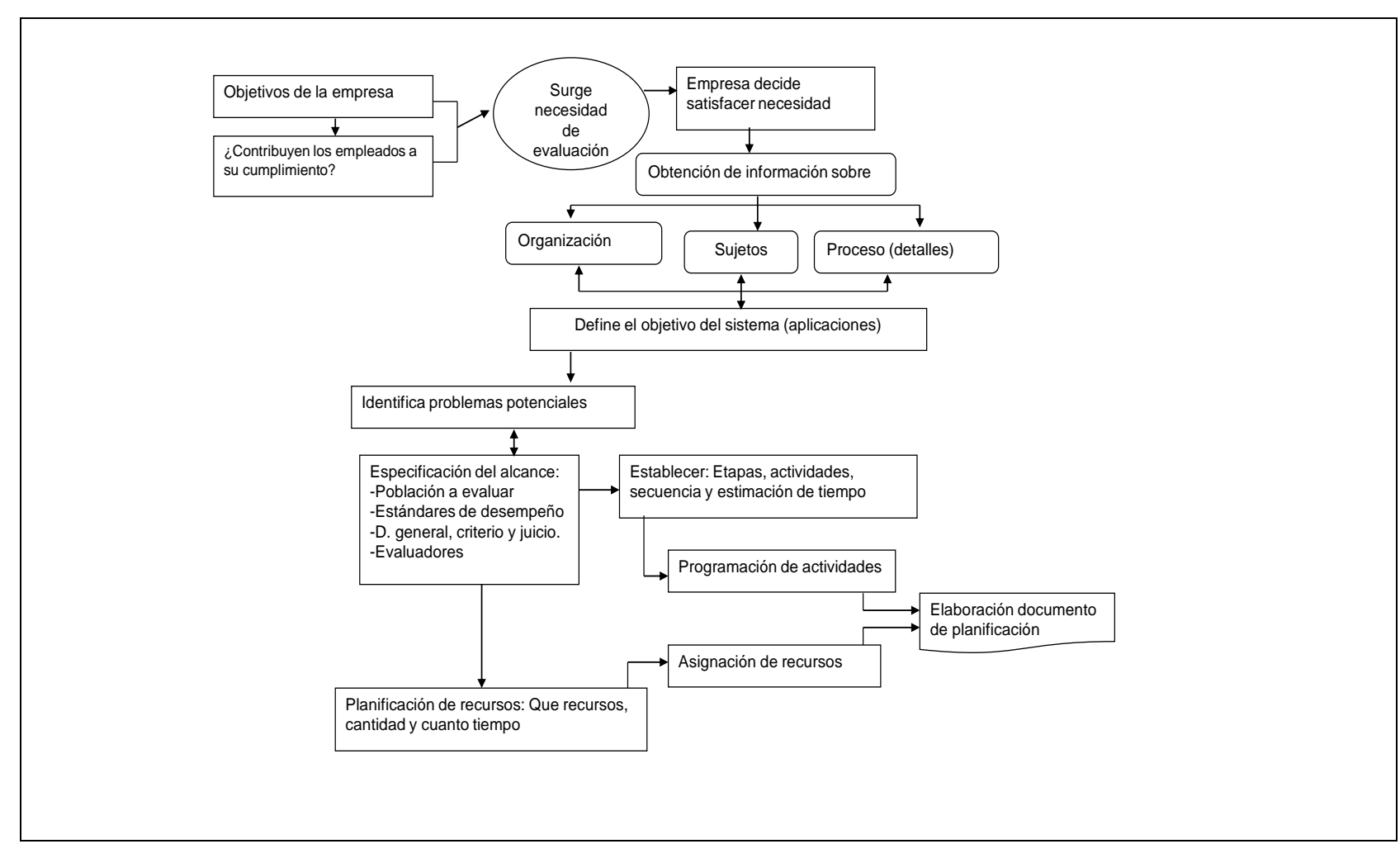

Fuente: Elaboración propia.

El registro de los datos se realizó por un equipo de auditoría, usando un formulario electrónico (Microsoft Office), diseñado a partir de lo señalado en el Programa de Auditoría, ver Tabla 1. Para disminuir el sesgo, el formulario electrónico, permite prevenir valores inexactos y fueron registrados por personas que no participaron del análisis. 
Tabla1: Atributos y procedimientos de auditoría

\begin{tabular}{|c|c|}
\hline ATRIBUTO & PROCEDIMIENTOS DE AUDITORÍA \\
\hline \multirow[t]{2}{*}{ 1. Definición del objetivo } & $\begin{array}{l}\text { a) Verificar si en el documento de la planificación existe la declaración específica del objetivo que se persigue con la implementación del proceso de } \\
\text { evaluación. }\end{array}$ \\
\hline & b) Consultar con los directivos y personal responsable cuál es el objetivo que persiguen al implementar el sistema y compararlo con el planificado. \\
\hline \multirow{6}{*}{ 2. Existencia } & a) Revisar la existencia de algún documento o archivo que contenga dicha planificación. \\
\hline & $\begin{array}{l}\text { b) Consultar con directivos y personal responsable respecto a la realización de una planificación previa al proceso. (Definición de qué se quería hacer, por } \\
\text { qué se quería hacer, utilización de recursos financieros y humanos, programación de actividades, etc.). }\end{array}$ \\
\hline & $\begin{array}{l}\text { c) Indagar con el personal directivo respecto a la previa definición y secuenciación de las etapas y actividades a través de las cuales se quiso desarrollar } \\
\text { el proceso. }\end{array}$ \\
\hline & $\begin{array}{l}\text { d) Verificar si junto con realizar una programación de actividades se designó a los responsables de realizarlas, ya sea a través de la revisión de documentos } \\
\text { u obteniendo confirmación con los responsables respecto a su designación anticipada. }\end{array}$ \\
\hline & $\begin{array}{l}\text { e) Revisar la existencia de un programa (o algo similar) que defina el tiempo que conllevará cada una de estas actividades, o en caso de que no exista, } \\
\text { realizar indagaciones con el personal para verificar que se estableció un período para llevar a cabo las diversas fases del proceso. }\end{array}$ \\
\hline & $\begin{array}{l}\text { f) Verificar a través de la revisión del documento de planificación o a través de indagaciones con el personal la existencia de una planificación de recursos, } \\
\text { así como una estimación del costo y luego la asignación de los mismos a través de un presupuesto. }\end{array}$ \\
\hline \multirow{4}{*}{ 3. Alineación } & $\begin{array}{l}\text { a) Evaluar la relación entre el objetivo declarado del sistema y los aspectos mencionados, a fin de determinar si dicho objetivo permite que el sistema sea } \\
\text { una herramienta que dirija el desempeño hacia el cumplimiento de los aspectos mencionados, es decir, misión, visión, estrategia y objetivos } \\
\text { organizacionales. }\end{array}$ \\
\hline & $\begin{array}{l}\text { b) Analizar la relación entre lo que la empresa desea evaluar, es decir, la dimensión general y los aspectos organizacionales mencionados, como misión, } \\
\text { visión, estrategia y objetivos, a fin de definir si efectivamente lo que se desea evaluar tiene directa relación con dichos aspectos. }\end{array}$ \\
\hline & $\begin{array}{l}\text { c) Analizar la relación entre lo que la empresa desea evaluar, es decir, la dimensión general, y los puestos sujetos a evaluación, a fin de definir si } \\
\text { efectivamente lo que se desea evaluar tiene directa relación con cada uno de los puestos a evaluar y con los estándares definidos para el cargo. }\end{array}$ \\
\hline & $\begin{array}{l}\text { d) Revisar los objetivos organizacionales y los estándares definidos de cada puesto, a fin de determinar si estos estándares están en función de lograr } \\
\text { dichos objetivos. }\end{array}$ \\
\hline \multirow[b]{2}{*}{ 4. Integridad } & $\begin{array}{l}\text { a) Revis ar el documento de planificación e identificar la población a evaluar y compararla con el total de trabajadores de la organización, a fin de verificar si } \\
\text { ambos concuerdan. }\end{array}$ \\
\hline & $\begin{array}{l}\text { b) Consultar con directivos cuál es la población que ellos desean evaluar y compararla con el total de trabajadores de la organización para verificar que se } \\
\text { han considerado todos los puestos. }\end{array}$ \\
\hline
\end{tabular}




\begin{tabular}{|c|c|}
\hline ATRIBUTO & PROCEDIMIENTOS DE AUDITORÍA \\
\hline \multirow{4}{*}{ 5. Cumplimiento } & $\begin{array}{l}\text { a) Indagar con el personal directivo si la realización de esta etapa del proceso se hizo en función de la previa definición y secuenciación de las actividades a } \\
\text { través de las cuales este se desarrollaría. }\end{array}$ \\
\hline & $\begin{array}{l}\text { b) Verificar que quienes se habían designado para llevar a cabo cada una de las actividades que involucra esta etapa del proceso, efectivamente hayan } \\
\text { realizado las labores que se les designaron. Esto es, a través de indagaciones con el personal y revisión de documentos, si procede, en donde se hubiera } \\
\text { podido registrar su participación. }\end{array}$ \\
\hline & $\begin{array}{l}\text { c) Verificar el cumplimiento del tiempo que se había establecido para realizar cada una de las actividades de esta etapa, ya sea mediante indagaciones con el } \\
\text { personal u otro tipo de revisiones que pudieran aportar evidencia respecto al tiempo utilizado para realizar cada una de las actividades. }\end{array}$ \\
\hline & d) Verificar los gastos incurridos en esta etapa. \\
\hline \multirow{2}{*}{ 6. Orientación } & a) Verificar si dentro de la planificación se consideran actividades de apoyo para el desempeño deficiente. \\
\hline & b) Verificar si dentro de la planificación se considera la realización de actividades o acciones dirigidas a fomentar el desempeño sobresaliente. \\
\hline \multirow[b]{6}{*}{ 7. Calidad } & a) Evaluar si las actividades realizadas en esta etapa del proceso fueron suficientes para llevarla a cabo. \\
\hline & b) Evaluar si los recursos asignados (tiempo y recursos financieros), para realizar la etapa de planificación fueron suficientes para realizarla a cabalidad. \\
\hline & $\begin{array}{l}\text { c) Indagar con el personal y posibles registros, respecto a las personas involucradas en la etapa de planificación y evaluar si el personal que se designó fue } \\
\text { suficiente. }\end{array}$ \\
\hline & d) Evaluar si las actividades (del proceso en su totalidad) definidas en la planificación son suficientes para cumplir el objetivo. \\
\hline & e) Evaluar si los recursos asignados al proceso total de evaluación son suficientes para conseguir el objetivo del sistema planteado. \\
\hline & f) Verificar que el cumplimiento del objetivo del sistema de evaluación está plas mado en la planificación. \\
\hline
\end{tabular}

Fuente: Elaboración propia, basado en Sánchez y Rojas (2014). 
Los datos analizados en esta investigación son de fuente primaria, recogidos mediante técnicas de análisis documental, la observación directa no participativa y el análisis de los procesos. De un total de 90 empresas evaluadas, se incluyeron 80, que cumplieron los criterios de inclusión, representando un 88,8\% del total. El criterio de exclusión dice relación con la obtención de evidencia de los procedimientos en su totalidad, en cada uno de los procesos. El análisis estadístico de los datos se realizó con el apoyo Minitab 16 y usó el Modelo de Regresión Múltiple.

\subsection{DEFINICIÓN DE VARIABLES}

- Variable dependiente - Evaluación, representa el resultado que se obtuvo en la auditoría realizada a la etapa de planificación del proceso de evaluación del desempeño y que se califica de la siguiente forma:

$1 \quad 0-57 \quad$ puntos - Muy malo

$2 \quad 58-72$ puntos - Regular

3 73-86 puntos - Bueno

4 87-100 puntos - Muy bueno

- Variables independientes: Definición del objetivo, Existencia, Alineación, Integridad, Cumplimiento, Orientación y Calidad.

1. Definición del objetivo

Significa que la organización tenga un objetivo, claramente definido que alcanzar a través de la evaluación de desempeño.

2. Existencia

Que la organización haya realizado una planificación del mecanismo de evaluación del desempeño, ya sea explícita o implícita.

3. Alineación

3.1. Que exista alineación entre aspectos organizacionales fundamentales, los objetivos de la evaluación del desempeño y lo que se desea evaluar.

a. Visión-misión-estrategia.

b. Objetivos organizacionales.

c. Con el puesto.

3.2. Significa que los estándares definidos para cada puesto contribuyan al cumplimiento de los objetivos organizacionales.

4. Integridad 
Que la planificación incluya la totalidad de los cargos de la organización, además de lo que espera en el desempeño de cada uno.

\section{Cumplimiento}

Que la planificación incluya mecanismos que permitan controlar y dirigir el proceso de evaluación del desempeño.
a. Etapas.
b. Actividades.
c. Tiempo.
d. Recursos financieros.
e. Personal involucrado.
6. Orientación

Que la planificación considere el desarrollo profesional basado en el cargo y la mejora en el desempeño.

\section{Calidad}

Significa que todos los componentes de la planificación estén dirigidos hacia la realización exitosa del proceso (tal como lo espera la organización) y al cumplimiento del objetivo del sistema de evaluación.

\section{RESULTADOS Y DISCUSIÓN}

En el contexto del análisis de la etapa "planificación del proceso de evaluación de desempeño de los trabajadores", las variables en estudio tienen una relación lineal y positiva, con excepción de integridad y orientación. El alfa de Cronbach general es de 0,8359. El valor es mayor que el valor de referencia común de 0,7 y sugiere que los elementos miden la misma construcción, ver Figura 2. 
Figura 2: Gráfica de matriz de análisis de elementos.

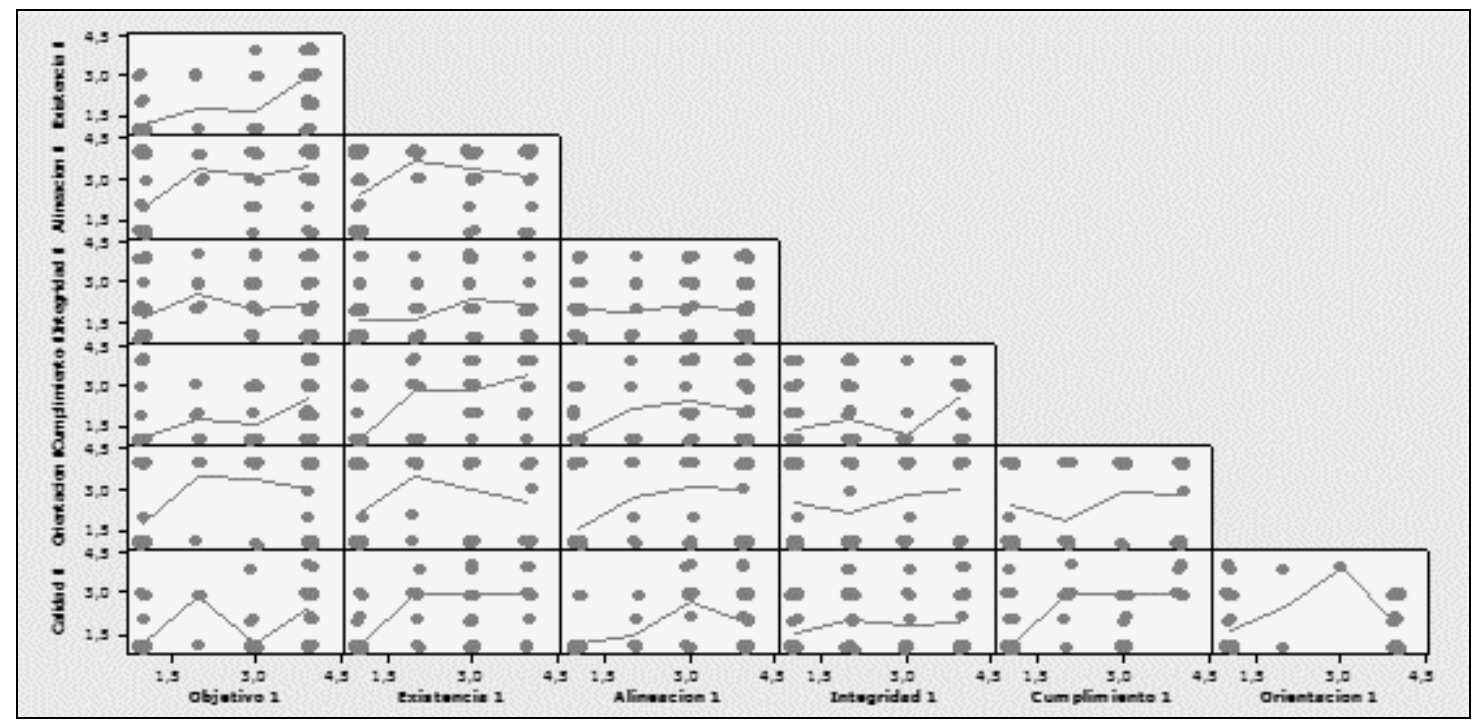

Fuente: Elaboración propia.

Según la tabla de las estadísticas de elementos omitidos se interpreta lo siguiente:

Para los datos sobre evaluación de las etapas de planificación del proceso de evaluación de desempeño de trabajadores:

La correlación ajustada de elementos, cuando las variables, Objetivo, Existencia, Cumplimiento, y Calidad se omiten del modelo es 0,$66 ; 0,67 ; 0,59 ; 0,54$, respectivamente. Sin embargo, cuando se omiten las variables Alineación, Integridad y Orientación la correlación ajustada de elementos es relativamente baja 0,43; 0,19 y 0,27 respectivamente, ver Tabla 2.

La correlación múltiple cuadrada cuando las variables Objetivo, Existencia, Cumplimiento y Calidad se omiten del modelo es 0,$55 ; 0,67 ; 0,59$ y 0,54 respectivamente. No obstante, cuando se omiten las variables Alineación, Integridad y Orientación, la correlación múltiple cuadrada es 0,$43 ; 0,19$ y 0,27 respectivamente, ver Tabla 2

El alfa de Cronbach cuando las variables Objetivo, Existencia, Cumplimiento y Calidad se omiten del modelo es 0,$67 ; 0,67 ; 0,69 ; 0,71$ respectivamente. Para las variables Alineación, Integridad y Orientación los valores difieren significativamente, 0,73; 0,77 y 0,77 respectivamente. Tabla 2

La correlación total ajustada de elementos y la correlación múltiple cuadrada son relativamente uniformemente y los valores de Alfa de Cronbach no difieren significativamente para las variables Objetivo, Existencia, Cumplimiento. En conjunto, la 
evidencia demuestra que las variables están relativamente correlacionadas, sin embargo, dicha correlación no es tan importante que amerite eliminar alguna variable explicativa.

Tabla 2: Estadística de elementos omitidos

\begin{tabular}{lcllll}
\hline Variable omitida & $\begin{array}{l}\text { Media total } \\
\text { Ajustada }\end{array}$ & $\begin{array}{l}\text { Desviación } \\
\text { estándar } \\
\text { total ajustada }\end{array}$ & $\begin{array}{l}\text { Correlación } \\
\text { total ajustada } \\
\text { por elementos }\end{array}$ & $\begin{array}{l}\text { Correlación } \\
\text { Múltiple } \\
\text { Cuadrada }\end{array}$ & $\begin{array}{l}\text { Alfa de } \\
\text { Cronbach }\end{array}$ \\
\hline Objetivos & 13,2 & 4,5 & 0,66 & 0,55 & 0,67 \\
Existencia & 13,8 & 4,6 & 0,67 & 0,68 & 0,67 \\
Alineación & 12,9 & 4,8 & 0,43 & 0,32 & 0,73 \\
Integridad & 13,7 & 5,1 & 0,19 & 0,08 & 0,77 \\
Cumplimiento & 13,8 & 4,7 & 0,59 & 0,52 & 0,69 \\
Orientación & 13,2 & 4,9 & 0,27 & 0,17 & 0,77 \\
Calidad & 13,9 & 4,8 & 0,54 & 0,49 & 0,71 \\
& & & & & \\
\hline
\end{tabular}

Fuente: Elaboración propia

Del análisis de cada una de las variables en forma separada se infiere que existe relación entre la variable dependiente "Evaluación" y las variables independientes: Objetivo, Existencia, Cumplimiento y Calidad. Por ello, se procede a medir la influencia simultánea e individual de todas las variables independientes sobre la variable "Evaluación”. Para ello se usó la regresión múltiple.

El modelo propuesto sobre la base del análisis de regresión múltiple es:

Evaluación $=-0,300+0,263$ Existencia + 0,169 Orientación + 0,272 Cumplimiento + 0,161 Objetivo $+0,173$ Calidad $+0,0893$ Integridad

Según el contraste de cada parámetro del modelo de regresión, medido a través de $\mathrm{t}$ de Student es bastante alto y lo p-valores para Existencia, Orientación y Cumplimiento son cero (0), para los parámetros de Objetivo, Calidad e Integridad son muy cercanos a cero (0), en tanto podemos rechazar la hipótesis nula y dichas variables deben incluirse en el modelo de regresión porque suministran información para explicar la variable dependiente “evaluación”, ver Tabla 3. 
Tabla 3: Coeficientes del Modelo Lineal

\begin{tabular}{lcccc}
\hline Variables & Coeficiente & SE Coeficiente & $\mathrm{T}$ & $\mathrm{P}$ \\
\hline Constante & $-0,2996$ & 0,1697 & $-1,77$ & 0,082 \\
Existencia & 0,26307 & 0,08005 & 3,29 & 0,002 \\
Orientación & 0,16905 & 0,03979 & 4,25 & 0,000 \\
Cumplimiento & 0,27168 & 0,06885 & 3,95 & 0,000 \\
Objetivo & 0,16060 & 0,05636 & 2,85 & 0,006 \\
Calidad & 0,17253 & 0,07273 & 2,37 & 0,020 \\
Integridad & 0,08934 & 0,05152 & 1,73 & 0,087 \\
& & & & \\
\hline
\end{tabular}

Fuente: Elaboración propia

Se considera que el modelo de regresión es bueno, teniendo presente que el $\mathrm{R}^{2}$ es 0,808 manteniéndose todo lo demás constante, además existen estudios que han definido el $\mathrm{R}^{2}$ igual a 0,8 o más en los modelos basados en datos de series temporales (Newbold, Carlson y Thorne, 2001). La diferencia entre $\mathrm{R}^{2}$ y $\hat{\mathrm{R}}^{2}$ no es grande, se infiere que las variables independientes irrelevantes provocan una pequeña reducción de la suma de los cuadrados de los errores. Tabla 4. 
Tabla 4: Estadísticos del Modelo

\begin{tabular}{lr}
\hline \multicolumn{1}{c}{ Estadísticos } & Resultado \\
\hline Se: Error Típico de Estimación & 0,489664 \\
R$^{2}$ : Coeficiente de determinación & 0,808 \\
R$^{2}$ : Coeficiente de determinación ajustado & 0,792 \\
S$^{2}$ : Varianza del error del modelo & 0,24 \\
SRC: Suma de los cuadrados de la regresión & 73,697 \\
SCE: Suma de los cuadrados de los errores & 17,503 \\
STC: Suma total de los cuadrados & 91,2 \\
n: Observaciones & 80 \\
K: Número de variables independientes & 6
\end{tabular}

Fuente: Elaboración propia

\section{CONCLUSIONES}

Se estudiaron 7 atributos que se cubrieron con la aplicación de 27 procedimientos de auditoría específicos, los cuales se orientaron a evaluar el proceso completo de la planificación de la evaluación del desempeño de los trabajadores.

Un aspecto preocupante a destacar es que se constató que ninguna de las 90 empresas revisadas, tuvo los 7 atributos bien evaluados, 5 de ellas tuvo un comportamiento muy bueno en sus atributos, en tanto que 49 empresas presentaron deficiencias en los atributos evaluados, lo más preocupante resulto ser que 35 empresas presentaron serias deficiencias en sus atributos y como agravante 5 de ellas presentaron dichas deficiencias en los 7 atributos evaluados.

A partir del análisis de correlación entre las variables explicativas: objetivo, existencia, alineación, integridad, cumplimiento, orientación y calidad, se infiere que dichas variables están correlacionadas entre ellas, no obstante, dicha correlación no es tan importante que amerite eliminar alguna variable explicativa.

Los resultados del análisis de la regresión lineal múltiple sugieren que, entre las variables con valor predictivo en la instancia de evaluar el resultado del proceso de planificación de la evaluación de desempeño de las personas son: 
o Que la organización haya realizado una planificación del mecanismo de evaluación del desempeño, ya sea explícita o implícita, denominado con la variable "existencia".

o Que la planificación considere el desarrollo profesional basado en el cargo y la mejora en el desempeño, denominado con la variable "orientación”.

o Que la planificación incluya mecanismos que permitan controlar y dirigir el proceso de evaluación del desempeño, denominado con la variable "cumplimiento".

o Que la organización tenga un objetivo, claramente definido que alcanzar a través de la evaluación de desempeño, denominado con la variable "objetivos".

o Que todos los componentes de la planificación estén dirigidos hacia la realización exitosa del proceso (tal como lo espera la organización) y al cumplimiento del objetivo del sistema de evaluación, denominado con la variable "calidad".

o Que la planificación incluya la totalidad de los cargos de la organización, además de lo que espera en el desempeño de cada uno, denominado con la variable "integridad"

Sin embargo, la variables "alineación”, que indica la existencia de alineación entre aspectos organizacionales fundamentales, los objetivos de la evaluación del desempeño y lo que se desea evaluar no tiene valor predictivo y por ello no se incorpora en el modelo.

\section{REFERENCIAS}

ALFARO DE PRADO, A. (1999). $\square$ El factor humano en las relaciones laborales. Manual de dirección y gestión. $\square$ Madrid: Ed. Pirámide.

ARENS, A.; \& LOEBBECKE, J. (1996). Auditoria: Un Enfoque Integral (6 ${ }^{a}$ Edición) .México: Prentice Hall Hispanoamericana.

ARENS, A.; ELDER, R.; \& BEASLEY, M. (2007). Auditoria: Un enfoque integral (11 ${ }^{a}$ Edición). México: Pearson Prentice Hall.

BOCK, L. (2015). La nueva fórmula del trabajo: Revelaciones de Google que cambiarán su forma de vivir y liderar. Madrid: Penguin Random House Grupo Editorial España.

ARIZA, J.; MORALES, A.; \& MORALES, E. (2004). Dirección y administración integrada de personas, fundamentos, procesos y técnicas en práctica. España: Mc Graw Hill.

BENTLEY, T. (1993). Capacitación empresarial. Santafé de Bogotá, Colombia: McGrawHill. 
COOPERS \& LYBRAND. (1984). Manual de Auditoria (3 ${ }^{a}$ Edición). Bilbao: Ediciones Deusto.

CHIAVENATO, I. (2007). Administración de recursos humanos. El capital humano de las organizaciones ( $8^{a}$ Edición). Colombia: McGraw Hill.

DE QUIJANO, S.; \& NAVARRO, J. (1999). Auditoría del sistema humano, los modelos de calidad y la evaluación organizativa. Revista de Psicología General y Aplicada. 52 (2), 301328

DESSLER, G.; \& VARELA, R. (2004). Administración de recursos humanos: un enfoque latinoamericano (2 ${ }^{a}$ Edición). México: Prentice Hall.

DOLAN, S.; SCHULER, R.; \& VALLE, R. (1999). La gestión de recursos humanos. España: Mc Graw Hill.

EXLEY, M.; \& LEGGE, K. (2001). Authority, Ambiguity, and Adaptation: The Personnel. Industrial Relations Journal, 6(3), 51- 65.

FERNÁNDEZ, G.; CUBIERO, J.; \& DALZIEL, M. (1996). Las competencias: clave para una gestión integrada de los recursos humanos (2 ${ }^{a}$ Edición). Bilbao: Ediciones Deusto

FONSECA, O. (2013). Sistemas de control interno. Lima, Ediciones IICO.

FOMBRUN, C.; TICHY, N.; \& DEVANNA, M. (1984). Strategic human resource management. New York: John Wiley and Sons.

GARCIA, J. (2003). Auditoría sociolaboral. Barcelona: Universitat Oberta de Catalunya.

GONZÁLEZ, O. (2010). Auditoría al sistema de compensaciones. Memoria para optar al título de Contador Público y Auditor, Escuela de Contador Público y Auditor, Universidad de Talca, Talca, Chile.

GÓMEZ-MEJÍA, L.; BALKIN, D.; \& CARDY, R. (2001). Dirección y gestión de recursos humanos ( $3^{a}$ Edición). España: Prentice Hall

GRUMAN, J.; \& SAKS, A. (2011). Performance management and employee engagement. Human Resource Management Review, 21, 123-136.

HERNÁNDEZ, D. (2007). Auditoría de gestión en el área de Recursos Humanos de ESSALUD Perú. Recuperado el 16 de septiembre del 2016 de http://www.gestiopolis.com/auditoria-de-gestion-en-el-area-de-recursos-humanos-deessalud-peru/

KEHOE, R.; \& WRIGHT, P. (2013). The Impact of High-Performance Human Resource Practices on Employees' Attitudes and Behaviors. Journal of Management, 2013, 39 (2), 366-391.

KLIKSBERG, B. (1998). Gerencia Pública para la Modernización del Estado. Revista Chilena de Administración Pública, 4 (19), 4-23. 
LUECKE, R. (2007). Gestión del desempeño. España: Harvard Business School Publishing Corporation, Ediciones Deusto.

MANJARRÉS, A.; CASTELL, R.; \& LUNA, C. (2013). Modelo de Evaluación del Desempeño Basado en Competencias. Ingeniare, (8) 11-29.

MARISTANY, J. (2000). Administración de recursos humanos. Argentina: Pearson Education.

MAUTZ, R. (1970). Fundamentos de Auditoría. Argentina: Macchi.

MEZA, M.; NARANJO, R.; \& PÉREZ, L. (2007). La evaluación del desempeño: herramienta vital en la dirección organizacional. Recuperado el 7 de agosto del 2016 de http://www.eumed.net/cursecon/ecolat/cu/2007/mame.htm

MILKOVICH, G.; \& BOUDREAU, J. (1994). Dirección y administración de recursos

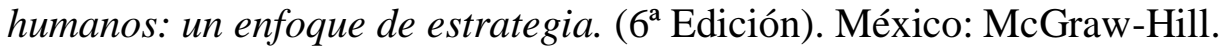

MONDY, M.; \& NOE, R. (2005). Administración de recursos humanos ( $9^{a}$ Edición). Mexico: Pearson Education.

NEVADO, D. (1998). La auditoría social de los recursos humanos como instrumento de gestión: futuro y limitaciones. Revista de Trabajo y Seguridad Social, (178), 175-230

NEWBOLD, P.; CARLSON, W.; \& THORNE, B. (2001). Estadística para administración y economía. España: Pearson Prentice Hall

PARENT, D.; SLOAN, N.; \& TSUCHIDA, A. (2015). Tendencias Globales en Capital Humano 2015: Liderando en el nuevo mundo del trabajo. Deloitte University Press.

PATTON, W. (1943). Manual del contador. México: UTEHA.

PEREDA, S.; BERROCAL, F.; \& LÓPEZ, M. (2004). Gestión de recursos humanos por competencias. Madrid, Editorial Centro de Estudios Ramón Areces S. A.

PEREDA, S.; BERROCAL, F.; \& LÓPEZ, M. (2002). Gestión de recursos humanos por competencias y gestión del conocimiento. Dirección y Organización, 5 (28). 43-54.

PÉREZ, R.; LEYVA C.; BAJUELO, A.; \& PÉREZ, T. (2015). La evaluación del desempeño individual, una herramienta para la toma de decisiones. Revista Correo Científico, 19 (4), 1-PÉREZ, E.; \& OREO, L., (2006). Manuales de dirección médica y gestión clínica. Madrid: España: Díaz de Santos.

PRICEWATERHOUSECOOPERS (2007). Marco Conceptual Metodológico. Enfoque de Auditoria PWC.

RAMLALL, S. J. (2006). Identifying and understanding HR competencies and their relationship to organizational practices. Applied H.R.M. Research, 11(1), 27 -38.

REYES, A. (2002). Relaciones humanas. I Parte. Administración de personal. México: Limusa. 
RODRÍGUEZ, M.; \& RAMÍREZ, P. (1997). Administración de la capacitación.México: McGraw Hill.

SALGADO, J. F.; \& CABAL, Á. L. (2011). Evaluación del desempeño en la Administración Pública del Principado de Asturias: Análisis de las propiedades psicométricas. Revista de Psicología del Trabajo y de las Organizaciones, 27(2), 75-91.

SÁNCHEZ, J.; PIZARRO, I.; ALVAREZ, A.; CASTILlO, M.; \& ALFARO, M. (2017). Auditoria a procesos de compensación en empresas de una economía emergente. Revista ENIAC PESQUISA, 6(2), 247-270

SÁNCHEZ. J.; \& ROJAS, O. (2014). Auditoría de recursos humanos. Madrid: Apyce.

SÁNCHEZ, J. (2013). Control de Gestión del Desempeño de los Recursos Humanos (2 ${ }^{a}$ Edición). Chile: Thomson Reuters.

SÁNCHEZ, J.; \& CALDERÓN, V. (2012). Diseño del proceso de evaluación del desempeño del personal y las principales tendencias que afectan su auditoría. Revista Pensamiento y Gestión. Colombia. Enero-Junio (32): 54-82.

SÁNCHEZ, J.; \& BUSTAMANTE, K. (2008). Auditoría al Proceso de Evaluación del Desempeño. Revista Contabilidad y Auditoría, (198), 105-133.

SASTRE, M.; \& AGUILAR, E. (2003). Dirección de Recursos Humanos, un enfoque estratégico. Madrid: McGraw Hill.

SIEGFRIED, H. (1965). Auditoria de Pessoal. RAE-Revista de Administracao de Empresas. $5(17), 149-168$.

SILVA, A.; OLIVEIRA, M.; ROSINI, A.; \& SILVA, G. (2016). Pesquisa de Clima Organizacional: ferramenta de modelo de Gestão na promoção de melhorias contínuas no Ambiente Institucional Financeiro. Revista ENIAC PESQUISA, 5(1), 19-28.

SLOSSE, C.; GIORDANO, S.; SERVIDEO, F.; DREISPIEL, G.; PACE, C.; \& DE MARCO, D. (1991). Auditoria: Un Enfoque Empresarial. Buenos Aires: Ediciones Machi.

TÉLLEZ, B. (2004). Auditoría un enfoque práctico. México, Thomson.

TRILLO HOLGADO, M. A. (2001). La evaluación del desempeño: nuevas tendencias e implicaciones. Capital Humano, 14(150), 66-72.

VALDÉS, M.; GARZA, R.; PÉREZ, I.; GÉ, M.; \& CHÁVEZ, A. (2015). Una propuesta para la evaluación del desempeño de los trabajadores apoyada en el uso de técnicas cuantitativas. Ingeniería Industrial, 36(1), 48-57.

VALLE, R.; \& WEISS, D. (1995). La Gestión estratégica de los recursos humanos. Delaware: Addison-Weasly Iberoamericana.

VALLE, R. (2004). La gestión estratégica de los recursos humanos (2 Edición). Madrid, España: Pearson Educación. 
WERTHER, W.; \& DAVIS, K (2008). Administración de recursos humanos, el capital humano de las empresas ( $6^{a}$ Edición). México: McGraw-Hill

WITTINGTON, O.; \& PANY, K. (2005). Principios de auditoría (14 ${ }^{a}$ Edición). México: McGraw-Hill Interamericana.

WRIGHT, P.; DUNFORD, B.; \& SNELL, S. (2001). Human resources and the resource based view of the firm. Journal of Management, 27, 701-721. 